\title{
Development of Percutaneous Endoscopic Lumbar Discectomy (PELD) Technology in China
}

Xifeng Zhang ${ }^{1}$, Jianwei $\mathrm{Du}^{2}$ and Anthony T Yeung ${ }^{3,4^{*}}$

${ }^{1}$ The Chinese General Hospital of PLA. No.28 Fuxing Road, Beijing 100853, China

${ }^{2}$ Yangzhou University affiliated Hospital. No.368 Hanjiang Road, Yangzhou, 225001, China

${ }^{3}$ University of New Mexico School of Medicine, Albuquerque, New Mexico

${ }^{4}$ Desert Institute for Spine Care, Phoenix, Arizona, USA

"Corresponding author: Yeung AT, Desert Institute for Spine Care, USA, Tel: +1 602-944-2900; E-mail: ayeung@sciatica.com

Rec Date: June 28, 2017; Acc Date: July 06, 2017; Pub Date: July 08, 2017

Copyright: (c) 2014 Yeung AT. This is an open-access article distributed under the terms of the Creative Commons Attribution License, which permits unrestricted use, distribution, and reproduction in any medium, provided the original author and source are credited.

\begin{abstract}
Introduction: The history and development of endoscopic spine surgery (PELD) in China is reviewed. Its significance and effect is predicted to have great implications for advancing spine care in China's working and aging population. Percutaneous spine technology was introduced simultaneously from Japan and the United states by Hijikata and Kambin in the early 1980s. It was called Arthroscopic Microdiscectomy (AMD). The access portal was called "Kambin's Triangle, with the discectomy technique through a "safe" puncture portal through an anatomical triangular zone in the foramen bordered by the facet and foraminal ligament dorsally, the exiting nerve ventrally, and the endplate of the caudal margin of the triangle.
\end{abstract}

Method: An expanded indication for the transforaminal Kambin technique was introduced to China by Anthony Yeung in 1998, and its evolution and contribution to modern minimally invasive spine care, called the Yeung Endoscopic Spine System (YESS ${ }^{\mathrm{TM}}$ ) technique, is reviewed. The percutaneous technique has been adopted by surgeons as well as non-surgeons who also undergo surgical training in their rehabilitation programs who integrate the technique through their various affiliations in China for painful degenerative conditions of the spine.

Results: The efficacy of the endoscopic technique has evolved significantly since Kambin, with additional contributions by Chinese surgeons and key opinion leaders of surgical and non-surgical Chinese associations that have co-existed, but are focused on providing treatment options for the Chinese population for centuries. Western Medicine has provided great influence on Chinese medicine, but a significant percentage of the Chinese population still cling to traditional treatment, embracing both Old and New methods. With respect to modern surgical techniques, surgeons and no surgeons are cooperative, and focus on results while working together. They recognize that with endoscopic spine surgery, it is important to maintain a success rate comparable to traditional western open surgery with less surgical morbidity utilizing the endoscope. Peer reviewed papers are emerging from China, using the EBM guidelines of Western Journals.

Conclusion: A rapidly increasing number of surgeries along with improving results will continue to drive this minimally invasive surgical method to China that bridges the gap between non-surgical pain management, physical medicine, and surgical intervention that focuses on the patho-anatomy and patho-physiology of spinal conditions of pain. This will be known as "surgical pain management, a term coined by Dr Anthony Yeung. Future developments will continue to drive the adoption of endoscopic surgery as a significant advancement for Chinese medicine and surgery.

\section{Keywords: Endoscopic spine surgery; Arthroscopic the past 10 years has flourished. This article chronicles the effect of the microdiscectomy \\ boom.}

\section{Effect of Endoscopic Spine Surgery in China}

Conservative treatment of low back pain has been a mainstay of Chinese medicine that traditionally incorporates Chinese massage, physical therapy, acupuncture, physical medicine, and other noninvasive and traditional Chinese exercise modalities. With the recent influence of Western culture and the increased exchange of Chinese and Western surgery techniques, Western procedures for treating low back pain has spread, and embraced by China. Percutaneous Endoscopic Lumbar Discectomy (PELD) technology in particular, in
PELD technology was introduced simultaneously from Japan and the United states by Hijikata and Kambin in the early 1980s. It was called Arthroscopic Microdiscectomy (AMD) [1,2]. The access portal was called "Kambin's Triangle, with the discectomy technique through a "safe" puncture fenestration through an anatomical triangular zone bordered by the facet and foraminal ligament dorsally, the exiting nerve ventrally and the endplate of the caudal margin of the triangle. The disc was entered through a cut - aspiration technique using coaxial fluoroscopic guidance. Dr. Tian, a Chinese surgeon treated LDH patients with a percutaneous non-coaxial AMD technique $[3,4]$. The technique, however, had not been not widely recognized and applied. 
In 1997 Dr. Anthony Yeung, by introducing a multi-channel flow integrated spine scope system with a working channel endoscope, advanced Kambin's transforaminal procedure. His coaxial endoscopic technique, with emphasis on endoscopic visualization of pathoanatomy, was introduced to China in 1998 at the 306 Military Hospital of the PLA in Beijing. Surgeons trained by Dr. Yeung first applied this technology, resulting in more than reported 200 cases reported in the Chinese literature with favorable minimally invasive endoscopic treatment outcomes. In 1998 Dr. Qiu in Taiwan followed Anthony Yeung's teaching. He visited Anthony Yeung to learn the technology and his "inside out" YESS technique [5]. Xifeng Zhang, at the 301 PLA hospital, gradually incorporated a variety of transforaminal as well as translaminar approaches to improve access to various patho-anatomy and by different angles transforaminally and, in order to overcome the technical difficulty of individual cases and to overcome the lack of specialized flexible pituitary forceps and flexible shavers utilized by Yeung. Essentially, the pathology was targeted through serial dilation and out-sides in trephining favored by the Thessys technique. Zhang also launched and studied the safety of the Far and Extreme lateral approach to the Lumbar spine, introduced by Ruetten that was presented at the SAS annual meeting in Berlin. Zhang emphasized the dangers of a pure extreme lateral approach published in spine by Ruetten that may result in a risk of injury to the abdominal and visceral organs if used indiscriminately without consideration of individual anatomic differences. He later utilized an endoscopic translaminar approach for lumbar the L5-S1 disc because it was more easily approached translaminarly. With this approach, he was able to carry out percutaneous endoscopic posterolateral fusion as well as intelaminar fusion. His PELD cases so far total 680 cases. Good/ Excellent results were obtained from a follow-up rate of $89.2 \%$. The technique spread outside Beijing after several Chinese conferences promoted and expanded the technology. PELD gained general recognition and acceptance as a standard of care. In April 2007 Dr. Yue Zhou organized in Chongqing, the "First Percutaneous Transforaminal International symposium" [6]. In June 2007, Dr. Guohua Lu held the Second National Conference on Minimally Invasive Spine Surgery in Changsha, also presenting the International Forum of endoscopic spinal surgery". In September 2008, the meeting was organized again in Chongqing, a "Third National Conference on Minimally Invasive Spine Surgery." In April 2010, the Beijing 301 Hospital organized the "2010 International Spine Surgery Minimally Invasive Spine Surgery Symposium with study courses" [7]. These meetings have caused PELD technology using both transforaminal and translaminar endoscopic techniques to become widely accepted in China.

Until recently, orthopedic spine surgeons performed most of the spine surgeries. Spine surgeons learned and also independently evolved the endoscopic technique since Kambin. With the spreading of this technique, not just spine surgeons now perform endoscopic spine surgery, but pain physicians, neurosurgeons, traditional Chinese medical doctors, and interventional doctors are adopting the technique. Some follow the Thessys technique of Hoogland, and others follow the YESS technique. Other systems followed, marrying Hoogland's outside-in technique while adopting a "targeted" technique that is dependent on accessing the patho-anatomy by whatever methods the surgeon feels most comfortable and familiar with. Many academic organizations were established, and conferences and study courses about endoscopic technique were held nearly every weekend in China.

The hospitals that adopted endoscopic spine surgeries developed from the first several hospitals to almost all levels and grades of hospitals in China and even some private clinics. More and more Chinese surgeons went abroad to participate in some international academic conferences about endoscopic spine surgeries and exchange experiences. In China, many local government health insurance began to cover parts of the cost of spinal endoscopic surgery, which also supported the development of this technology. The cost of endoscopic spine surgery was different in different hospitals. And the average cost is between $\$ 3000$ to $\$ 4000$ USD [8]. The minimum can be as low as $\$ 1500$, and the maximum is not more than $\$ 7000$.

An estimated number of spine surgeries in China in a year are 500,000-600,000, of which 50,000-60,000 are endoscopic surgeries. Although it is only about $10 \%$ of all spine surgeries, this proportion continues to increase rapidly each year. In 2010, less than 30 surgeons performed endoscopic spine surgery by few doctors who can finish more than 100 cases in a year. In 2016, nearly 200-300 doctors perform endoscopic spine surgery with more than 100 surgeons able to successfully complete more than 100 cases /year. Some surgeons have finished more than 500 cases. Due to its sheer size, China may soon, if not already, be the country with the largest number of cases performed each year.

The indications for surgery are constantly being expanded with the development of new equipment,along with evolving surgical skills and surgical techniques. The conditions treated range from discogenic pain to contained lumbar disc herniation to migrated disc herniation, calcified prominent annular protrusions, foraminal and canal spinal stenosis [8]. All degenerative conditions are now being treated endoscopically. In the early stages, endoscopic spine surgery was used only in the lumbar spine, but in the resent years endoscopic spine surgery is successfully used in the cervical spine and thoracic spine [9]. This technique is now also utilized not just for degenerative spinal diseases, but spinal tuberculosis, infectious disease, spinal fractures, and spinal tumors [10].

Thanks to Dr. Anthony Yeung's introduction of his endoscopic technical inventions, endoscopic endoscope design, his creation of selective endoscopic philosophy ${ }^{\mathrm{TM}}$ [11], and his expansion of the technique, along with his perseverance of teaching and promotion in China, endoscopic MIS surgery is becoming mainstream. The current technology brings proficiency and greater standardization of the technique so that surgeries can be performed earlier in the lumbar disease process for discogenic pain, contained herniations and annular tears, called selective endoscopic discectomy ${ }^{\mathrm{TM}}$ and thermal annuloplasty. Other exciting modern advances, such as Biologics and Robotics, are ideal for incorporation by endoscopy and currently embraced and developed by Dr. Yeung [12]. Most surgeries can be completed within 1 hour. Speed may be a factor to avoid prolonged traction and irritation of sensitive nerves such as the dorsal root ganglion. Advances such as artificial intelligence for robotics application under development by Dr. Yeung will help standardize endoscopic spine surgery to the extent that is may become a new standard on MIS spine care. Dr. Xifeng Zhang, due to the simplicity of many conditions treated, has, in 1 day, performed up to 16 operations [13]. Because of the many advantages of endoscopic techniques, incorporating simplicity, less surgical morbidity, rapid recovery, and a minimal complication rate at least comparable but even less when compared to open surgery, the technology is being increasingly welcomed by Chinese doctors. In just a few years, the country now has hundreds of hospitals with endoscopic equipment [14]. We believe this technology is ideal for China, as it will reach more people who are currently underserved. The improving results will continue to drive 
this minimally invasive surgical method that will bridge the gap between interventional pain management [15], spine rehabilitation and surgical intervention that focuses on the patho-anatomy and patho-physiology of spinal conditions of pain. The Chinese Medical Association has developed interest in incorporating this technology under its umbrella. Public/private joint partnerships for minimally invasive spine care are being developed $[16,17]$.

\section{Acknowledgments}

Dr. Xifeng Zhang and Jianwei Du, who trained with Dr. Yeung in Phoenix, are now, in turn, training Chinese doctors. Dr. Bin Zhu, from Peking University Third Hospital (PUTH) the largest spine hospital in China, has performed $>500$ spine cases/year. Dr. Ding Yu, Md. PhD. a professor Rehabilitation Medicine of the Navy General Hospital in Beijing Is forming a committee, The Chinese Association of Chinese Medicine (CACM), a branch of the Chinese Medical Association, currently acting as their "Honorary Director". Dr. Xifeng Zhang is a consultant to Dr. Yu. And Dr. Bin Zhu recently reported complications from PELD discussion groups in China, is emphasizing proper training for all providers, surgeon and non-surgeon alike who will embrace the responsibility to make this evolving minimally invasive procedure safe, cost effective, and beneficial to the vast population of China that focus on following the results, as opposed to the money, a major focus in Western Societies. Dr. Yeung provides his surgical philosophy and technique of the "YESS" technique and system for China, and is continuing his efforts to bring endoscopic surgery to China through the Chinese Medical Association as well as through Surgical Organizations.

\section{References}

1. Hijikata S (1989) Percutaeous nucleotomy: A new concept technique and 12 years' experience. Clin Othop 238: 9-23.

2. Kambin P, Zhou L (1996) History and current status of percutaneous arthroscopic disc surgery. Spine (Phila Pa 1976) 21: 57S-61S.

3. Tian SJ, Wang JJ, Liu DL, (1993) PELD primary report. Chinese Journal of Orthopaedics 13: 3-7.

4. Tian SJ (1997) PELD. Chinese Journal of Orthopaedics 17: 321-323.
5. Zou DW, Ma HS, Hai Y, (1998) Spine endoscopy for lumber disc herniation. (add:80 cases primary report). Chinese Journal of Spine and Spinal Cord 8: 307-310.

6. Zhang XF, Wang Y, Xiao SH, Liu ZS, Liu BW, et al. (2007) Percutaneous treatment of poster-lateral approach for far lateral disc herniation. The Journal of Practical Medicine 23: 3479-3480.

7. Zhang XF, Wang Y, Xiao SH (2010) Endoscopic treatment of different approaches L5-S1 disc herniation. Chinese Journal of Orthopaedics 30; 341-345.

8. Du J, Tang X, Jing X, Li N, Wang Y, et al. (2016) Outcomes of percutaneous endoscopic lumbar discectomy via a translaminar approach, especially for soft, highly down-migrated lumbar disc herniation. International Orthopaedics 40: 1247-1252.

9. Yeung A, Tsou PM (2002) Posterolateral endoscopic excision for lumbar disc herniation : Surgical technique, outcome, and complications in 307 consecutive cases. Spine 27: 722-731.

10. Bao L, Hai Y, Ma HS, Zhou XF, Zou DW, et al. (2005) Endoscopic lumbar discectomy spine surgery Long-term effect. Orthopedic Journal of China 13: 184-186.

11. Yeung AT (2016) Endoscopic decompression, foraminalplasty and dorsal rhizotomy for foraminal stenosis and lumbar spondylosis: A hybrid procedure in lieu of fusion. J Neurol Disord 4: 322.

12. Yeung AT (2017) Robotics in the MIS spine surgery arena: A new role to advance the adoption of endoscopic surgery as the least invasive spine surgery procedure. J Spine 6: 374.

13. Zhang XF, Wang Y, Xiao ZH, Liu ZS, Liu BW, et al. (2006) Lateral approach for percutaneous endoscopic discectomy in the feasibility and clinical application. Chinese Journal of Spine and Spinal Cord 16: 659-662.

14. Wu XC, Zhou Y (2009) Spinal endoscopic lumbar discectomy: Technical progress and prospects. Chinese Journal of Minimally Surgery 9: 283-285.

15. Xu SK, Tong RN, Tong RL (2009) Percutaneous endoscopic surgery for lumbar disc disease of the efficacy analysis. Chinese Journal of Spine and Spinal Cord 19: 330-335.

16. Yeung AT (2016) Intradiscal therapy and transforaminal endoscopic decompression: Opportunities and challenges for the future. JNeurol Disord 4: 303.

17. Yeung AT (2017) Transforaminal endoscopic decompression for painful degenerative conditions of the lumbar spine: A review of one surgeon's experience with over 10,000 cases since 1991. J Spine Neurosurg 6: 2. 